AJ, in press

\title{
Optical, Infrared, and Ultraviolet Observations of the $X$-Ray Flash XRF 050416A
}

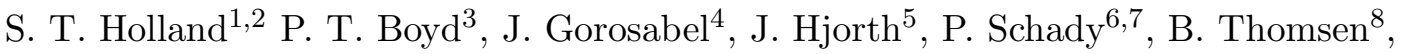 \\ T. Augusteijn ${ }^{9}$, A. J. Blustin ${ }^{6}$, A. Breeveld ${ }^{6}$, M. De Pasquale ${ }^{6}$, J. P. U. Fynbo ${ }^{5}$, N. Gehrels ${ }^{3}$,

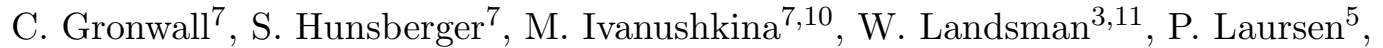 \\ K. McGowan ${ }^{6,12}$, V. Mangano ${ }^{13}$, C. B. Markwardt ${ }^{3}$, F. Marshall ${ }^{3}$, K. O. Mason ${ }^{6,14}$, A. Moretti ${ }^{15}$, \\ M. J. Page ${ }^{6}$, T. Poole ${ }^{6}$, P. Roming ${ }^{7}$, S. Rosen ${ }^{6}, \&$ M. Still ${ }^{2,3,16}$
}

\begin{abstract}
We present ultraviolet, optical, and infrared photometry of the afterglow of the $X$ ray flash XRF 050416A taken between approximately 100 seconds and 36 days after the burst. We find an intrinsic spectral slope between $1930 \AA$ and $22200 \AA$ of $\beta=$
\end{abstract}

\footnotetext{
${ }^{1}$ Code 660.1, NASA's Goddard Space Flight Centre, Greenbelt, MD 20771, U.S.A.

sholland@milkyway.gsfc.nasa.gov
}

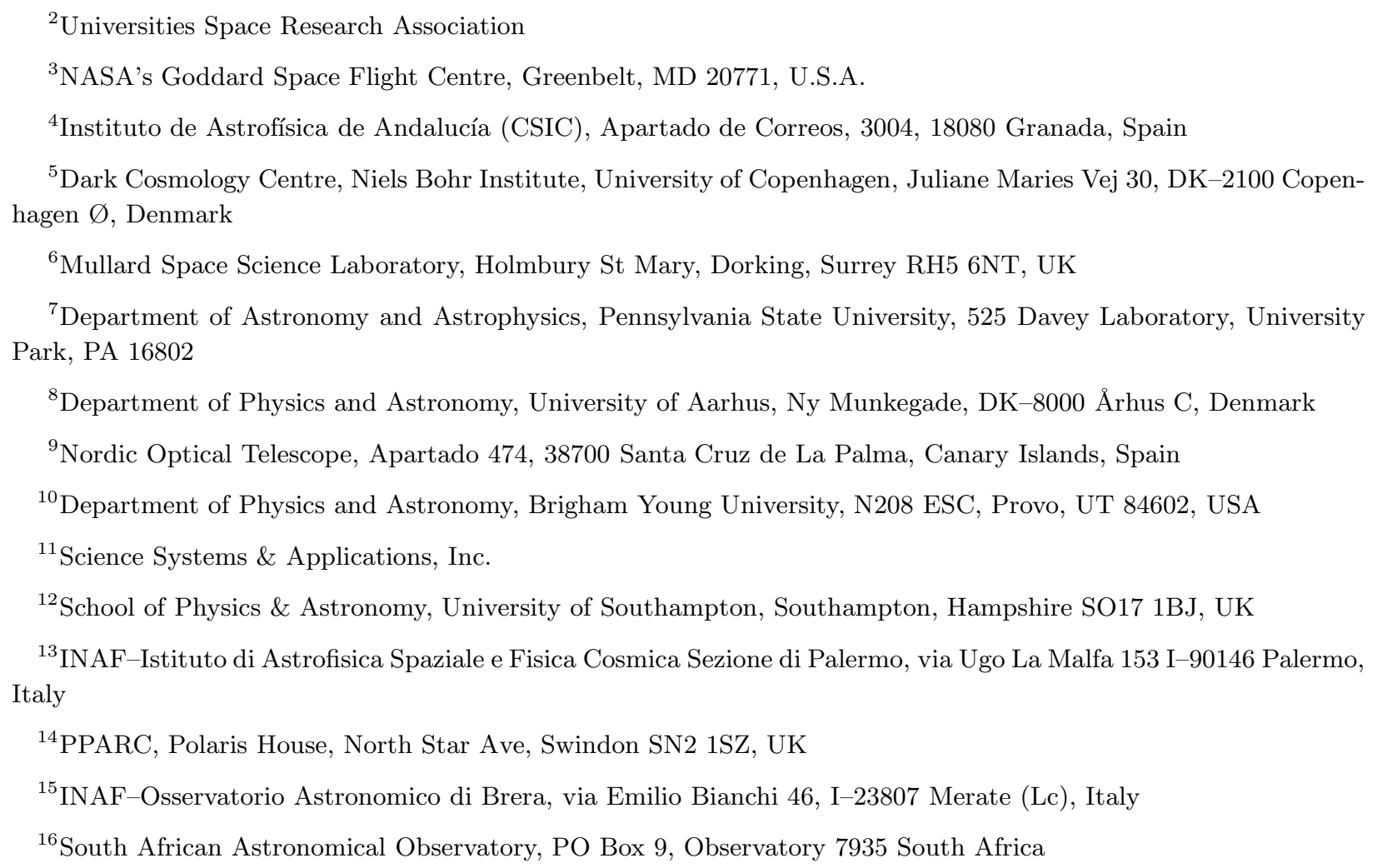


$-1.14 \pm 0.20$ and a decay rate of $\alpha=-0.86 \pm 0.15$. There is no evidence for a change in the decay rate between approximately 0.7 and 4.7 days after the burst. Our data implies that there is no spectral break between the optical and $X$-ray bands between 0.7 and 4.7 days after the burst, and is consistent with the cooling break being redward of the $K_{s}$ band $(22200 \AA)$ at 0.7 days. The combined ultraviolet/optical/infrared spectral energy distribution shows no evidence for a significant amount of extinction in the host galaxy along the line of sight to XRF 050416A. Our data suggest that the extragalactic extinction along the line of sight to the burst is only approximately $A_{V}=0.2 \mathrm{mag}$, which is significantly less than the extinction expected from the hydrogen column density inferred from $X$-ray observations of XRF 050416A assuming a dust-to-gas ratio similar to what is found for the Milky Way. The observed extinction, however, is consistent with the dust-to-gas ratio seen in the Small Magellanic Cloud. We suggest that XRF 050416A may have a two-component jet similar to what has been proposed for GRB 030329. If this is the case the lack of an observed jet break between 0.7 and 42 days is an illusion due to emission from the wide jet dominating the afterglow after approximately 1.5 days.

Subject headings: gamma rays: bursts

\section{Introduction}

The Swift satellite (Gehrels et al. 2004) is a multi-instrument observatory designed to detect and rapidly localize gamma-ray bursts (GRBs). The observatory contains three instruments. The Burst Alert Telescope (BAT; Barthelmy et al. 2005) is used to identify GRBs and localize them to $\lesssim 3^{\prime}$ in the energy range $15-150 \mathrm{keV}$. Once a burst has been detected Swift slews to point its two narrow-field instruments, the $X$-Ray Telescope (XRT; Burrows et al. 2005), and the UltraViolet/Optical Telescope (UVOT; Roming et al. 2005) at the burst. The XRT obtains $X$-ray localizations to $\lesssim 5^{\prime \prime}$ in the energy range $0.2-10 \mathrm{keV}$. The UVOT simultaneously obtains localizations to $\approx 0.5$ then cycles through a set of optical and ultraviolet filters.

The $X$-ray flash (XRF) XRF 050416A was detected in the constellation Coma Berenices by the BAT at 11:04:44.5 UTC (Sakamoto et al. 2005a). The gamma-ray light curve showed a slowly rising peak followed by several smaller peaks. The burst had a $T_{90}$ duration of only $2.4 \pm 0.2 \mathrm{~s}$ in the $15-150$ $\mathrm{keV}$ band. Sakamoto et al. (2006) find a peak energy of $E_{p}=15.6_{-2.7}^{+2.3} \mathrm{keV}$, making XRF 050416A a soft burst, and they classify XRF $050416 \mathrm{~A}$ as an XRF. The fluence was $(3.5 \pm 0.3) \times 10^{-7} \mathrm{erg}$ $\mathrm{cm}^{-2}$ in the $15-150 \mathrm{keV}$ band (Sakamoto et al. 2006). The fluence in the soft $15-25 \mathrm{keV}$ band $\left((1.7 \pm 0.2) \times 10^{-7} \mathrm{erg} \mathrm{cm}^{-2}\right)$ was larger than that in the harder $50-100 \mathrm{keV}$ band $\left(3.4_{-0.6}^{+1.0} \times 10^{-8}\right.$ erg $\mathrm{cm}^{-2}$ ). This, and the low peak energy, makes XRF 050416A an XRF. A detailed analysis of the gamma-ray properties of XRF 050416A is presented in Sakamoto et al. (2006).

An $X$-ray afterglow was identified by Kennea et al. (2005) and is discussed in detail in Mangano 
et al. (2006). The $X$-ray light curve follows the canonical $X$-ray light curve described by Nousek et al. (2006) with an early-time decay of $\alpha_{X}=-2.4 \pm 0.5$ out to $172 \mathrm{~s}$, where the flux density, $f_{\nu}$, is related to the time since the BAT trigger by $f_{\nu}(t) \propto t^{\alpha}$. The canonical light curve has an initially steep decay due to curvature effects in the relativistic fireball. This is followed by a slow decay due to energy injection. The light curve makes a second transition to a decay slope of approximately -1 when energy injection ends. The slow decay section for XRF 050416A has a slope of $\alpha_{X}=-0.44 \pm 0.13$ and lasts from 172 to $1450 \mathrm{~s}$. The late-time decay has a slope of $\alpha_{X}=-0.88 \pm 0.02$, which continues until at least 42 days after the BAT trigger. The $X$-ray spectrum after $1450 \mathrm{~s}$ has a slope of $\beta_{X}=-1.04_{-0.11}^{+0.05}$, where the relationship between flux density and frequency is $f_{\nu} \propto \nu^{\beta}$, and shows evidence for a hydrogen column density in the host galaxy of $N_{H}=6.8_{-1.2}^{+1.0} \times 10^{21} \mathrm{~cm}^{-2}$. If we assume the Predehl \& Schmitt (1995) relationship between hydrogen column density and extinction in the Milky Way, $N_{\mathrm{H}}=\left(1.79 \times 10^{21}\right) A_{V}$, then this column density implies $A_{V}=3.8_{-0.7}^{+0.6}$ in the host galaxy along the line of sight to XRF 050416A.

The optical afterglow was first identified by Cenko \& Fox (2005) using the Palomar 200-inch Hale Telescope and confirmed by Anderson et al. (2005) using the ANU 2.3-m telescope. Fox (2005) noted that the afterglow is visible in the UVOT's UVW2 filter and stated that this indicates a redshift of $z \lesssim 1$. A spectrum of the host galaxy was obtained 51 days after the burst by Cenko et al. (2005). They measured a redshift for the host of $z=0.6535 \pm 0.0002$ from [OII], $\mathrm{H} \beta, \mathrm{H} \gamma$, and $\mathrm{H} \delta$ emission lines. The host's blue colour suggests ongoing star formation, as is seen in many GRB host galaxies. UVOT magnitudes, based on preliminary photometric calibrations, were published by Schady et al. (2005a,b). This paper supersedes those results. Soderberg et al. (2005) found a $4.86 \mathrm{GHz}$ radio afterglow with a flux density of $260 \pm 55 \mu \mathrm{Jy}$ approximately 5.6 days after the BAT trigger.

Excluding the short-hard bursts (Kouveliotou et al. 1993) XRF 050416A, with a redshift of 0.6535, is one of the nearest of the Swift-detected GRBs with spectral redshift determinations. Its $T_{90}$ duration and $E_{p}$ are similar to those of the XRF 050406 (Krimm et al. 2005; Schady et al. 2006) and are fairly typical of XRFs in general. Sakamoto et al. (2006) have shown that XRF 050416A is consistent with the relationship between the isotropic energy equivalent, $E_{\text {iso }}$, and the peak energy in the rest frame, $E_{p}^{\prime 1}$, found by Amati et al. (2002) for classical long-soft GRBs. This suggests that XRFs and GRBs are intimately related. XRF 050416A does not, however, follow the the empirical relation between $E_{p}^{\prime}, E_{\text {iso }}$, and the jet break time in the rest frame, $t_{j}^{\prime}$ of Liang \& Zhang (2005), which suggests that either this XRF has an unusually late jet break or that something is preventing us from seeing the jet break, such as a long-lasting emission component. Something similar was seen in GRB 021004, where identifying the jet break was complicated by several emission components (Holland et al. 2003).

In this paper we present space- and ground-based ultraviolet, optical, and infrared observations of XRF 050416A. We have adopted a cosmology with a Hubble parameter of $H_{0}=70 \mathrm{~km} \mathrm{~s}^{-1} \mathrm{Mpc}^{-1}$,

\footnotetext{
${ }^{1}$ Throughout this paper we use primes to indicate quantities in the rest frame.
} 
a matter density of $\Omega_{m}=0.3$, and a cosmological constant of $\Omega_{\Lambda}=0.7$. For this cosmology a redshift of $z=0.6535$ corresponds to a luminosity distance of $3917 \mathrm{Mpc}$ and a distance modulus of 42.96. One arcsecond corresponds to 11.48 comoving kpc, or 6.95 proper kpc. The look back time is 6.05 Gyr.

\section{Observations}

The optical afterglow for XRF 050416A is located at R.A. $=12: 33: 54.6$, Dec. $=+21: 03: 27$ (J2000) (Cenko \& Fox 2005), which corresponds to Galactic coordinates of $\left(b^{\mathrm{II}}, l^{\mathrm{II}}\right)=\left(+82^{\circ} .7316,268^{\circ} .7316\right)$. The reddening maps of Schlegel et al. (1998) give a Galactic reddening of $E_{B-V}=0.03 \pm 0.02 \mathrm{mag}$ in this direction. The corresponding Galactic extinctions are $A_{U}=0.16, A_{B}=0.13, A_{V}=0.10$, $A_{R_{C}}=0.08, A_{I_{C}}=0.06$, and $A_{K_{s}}=0.01$. The Galactic extinctions in the UVOT ultraviolet filters were calculated using the Milky Way extinction law from Pei (1992). The ultraviolet extinctions are $A_{\mathrm{UVW} 1}=0.23, A_{\mathrm{UVM} 2}=0.29$, and $A_{\mathrm{UVW} 2}=0.21$.

The field of XRF 050416A is shown in Figure 1.

\subsection{UVOT Data}

The Swift spacecraft slewed promptly when the BAT detected XRF 050416A, and UVOT began imaging the field $65 \mathrm{~s}$ after the BAT trigger. A range of 14-16 exposures were taken in each UVOT filter between 11:05:49 UTC on 16 Apr 2005 and 03:45:46 UTC on 18 Apr 2005. The UVOT has $U B V$ filters which approximate the Johnson system, and three ultraviolet filters: UVW1 with a central wavelength of $\lambda_{0}=2600 \AA$, UVM2 with $\lambda_{0}=2200 \AA$, and UVW2 with $\lambda_{0}=1930 \AA$. Examination of the first settled observation (a $100 \mathrm{~s}$ full-frame exposure in $V$ ) revealed a new source relative to the Digital Sky Survey inside the XRT error circle. This source had a magnitude of $V=19.19 \pm 0.29$. The UVOT position of the source is R.A. $=12: 33: 54.596$, Dec. $=+21: 03: 26.07$ (J2000) with a statistical error of $0{ }^{\prime \prime} 01$ and an absolute astrometric accuracy of 0.56 (90\% containment). This position is 0 " 3 from the reported XRT position (Mangano et al. 2006). It is inside the XRT error circle and consistent with the ground-based detection reported by (Cenko \& Fox 2005). Subsequent exposures showed the source to be rapidly fading.

The UVOT took 342 exposures of the field containing XRF 050416A between 2005 Apr 16 and 2005 May 13 UTC. For the vast majority of these exposures the afterglow was too faint to be detected. The UVOT photometry that was used in this paper is shown in Figures 2, 3, and 4 and presented in Table 1. Magnitudes have not been corrected for extinction. All upper limits reported in Table 1 are $3 \sigma$ and were obtained using a $2^{\prime \prime}$ radius aperture with aperture corrections applied as discussed below. 


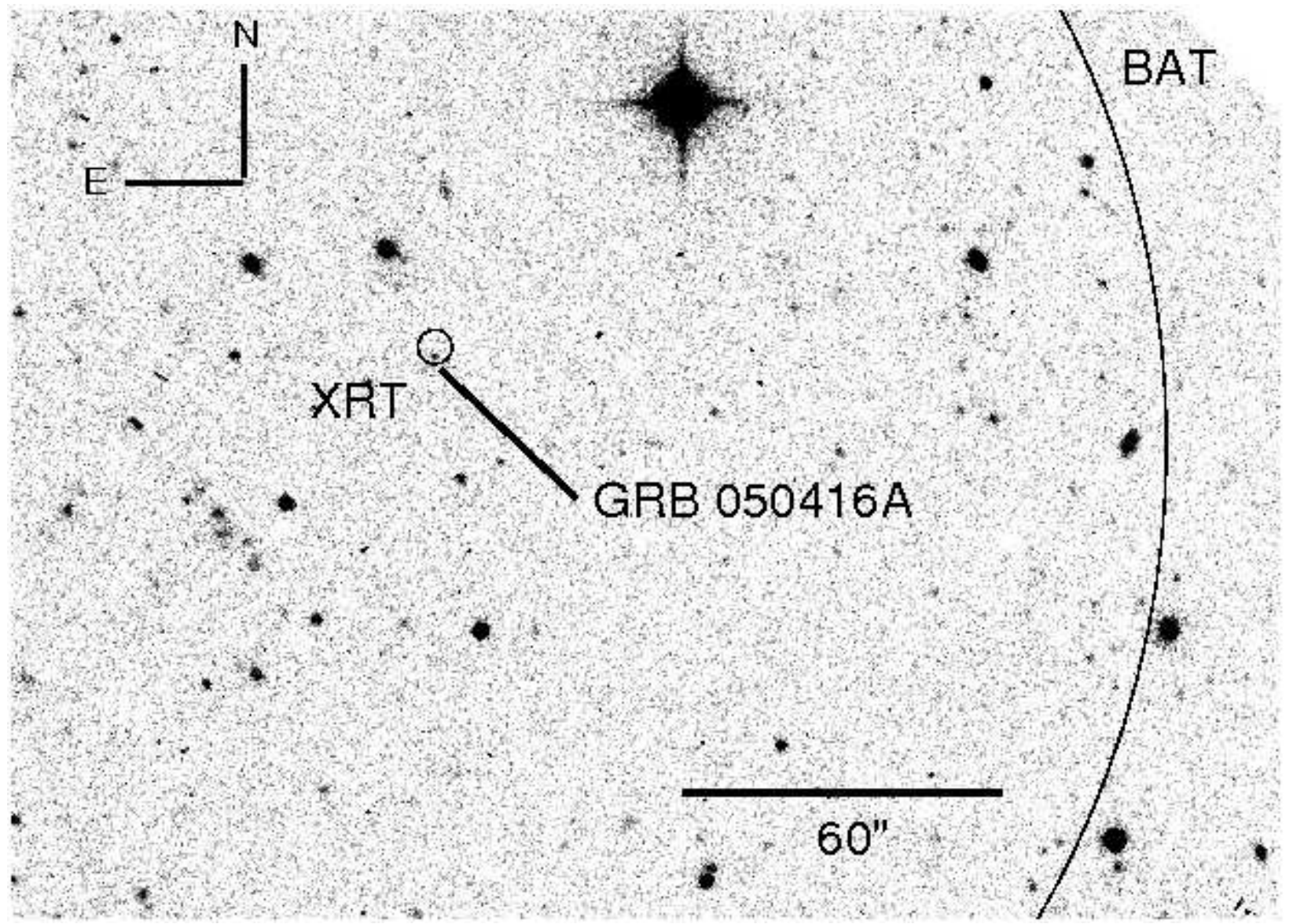

Fig. 1. - This figure shows the $2 \times 1200$ second DFOSC $V$-band image taken 60415 seconds after the BAT trigger. The large circle is the $3^{\prime}$ radius BAT error circle (Sakamoto et al. 2005b). The small circle is the 3.' 3 radius XRT error circle (Mangano et al. 2006). North is up and east is to the left. 


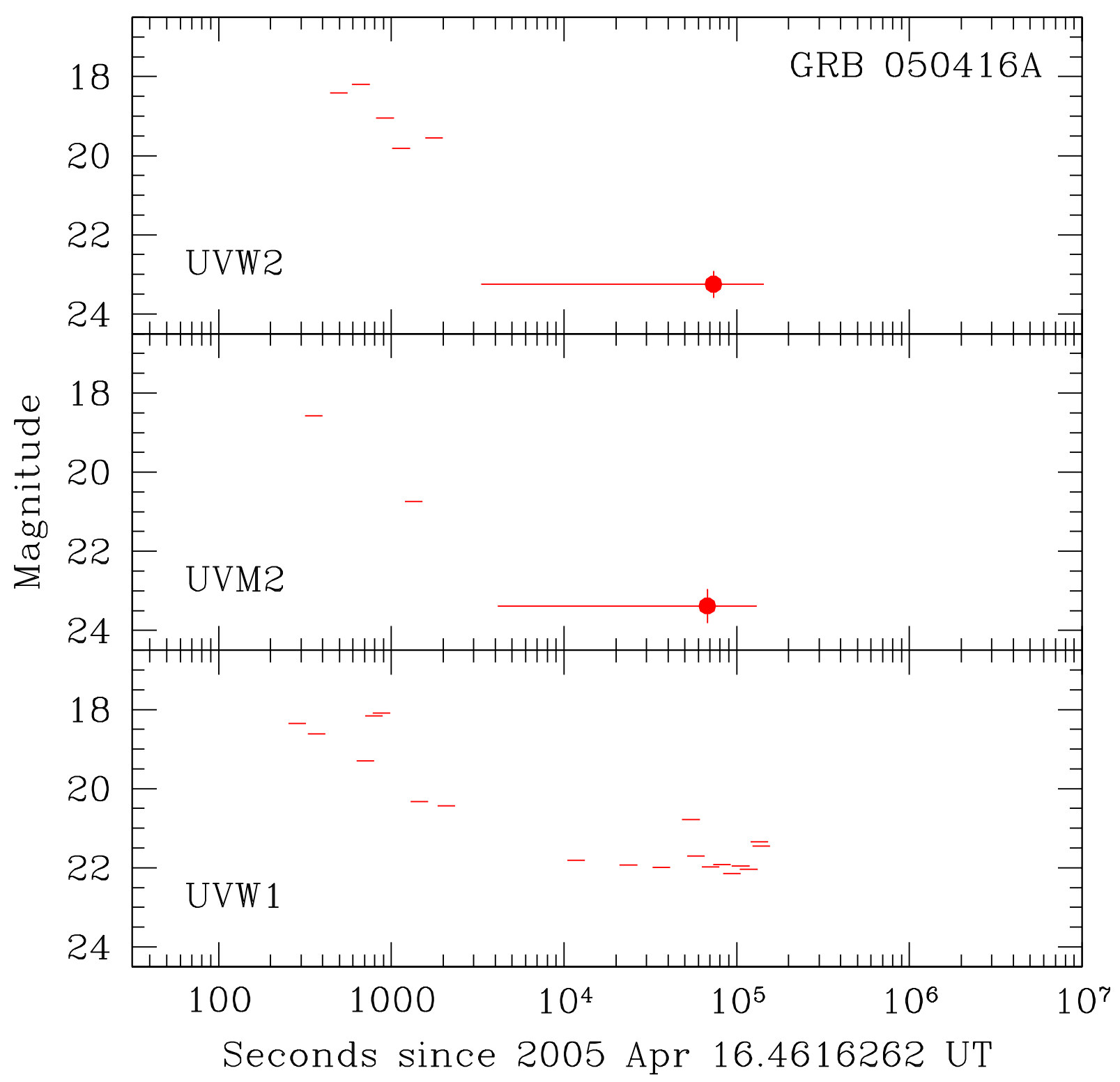

Fig. 2.- This Figure shows the UVW2, UVM2, and UVW1 photometry of the afterglow of XRF 050416A. Red circles indicates UVOT detections. Upper limits are represented by horizontal lines. The horizontal error bars on the detections indicate the total period during which data was collected, not the exposure time. 


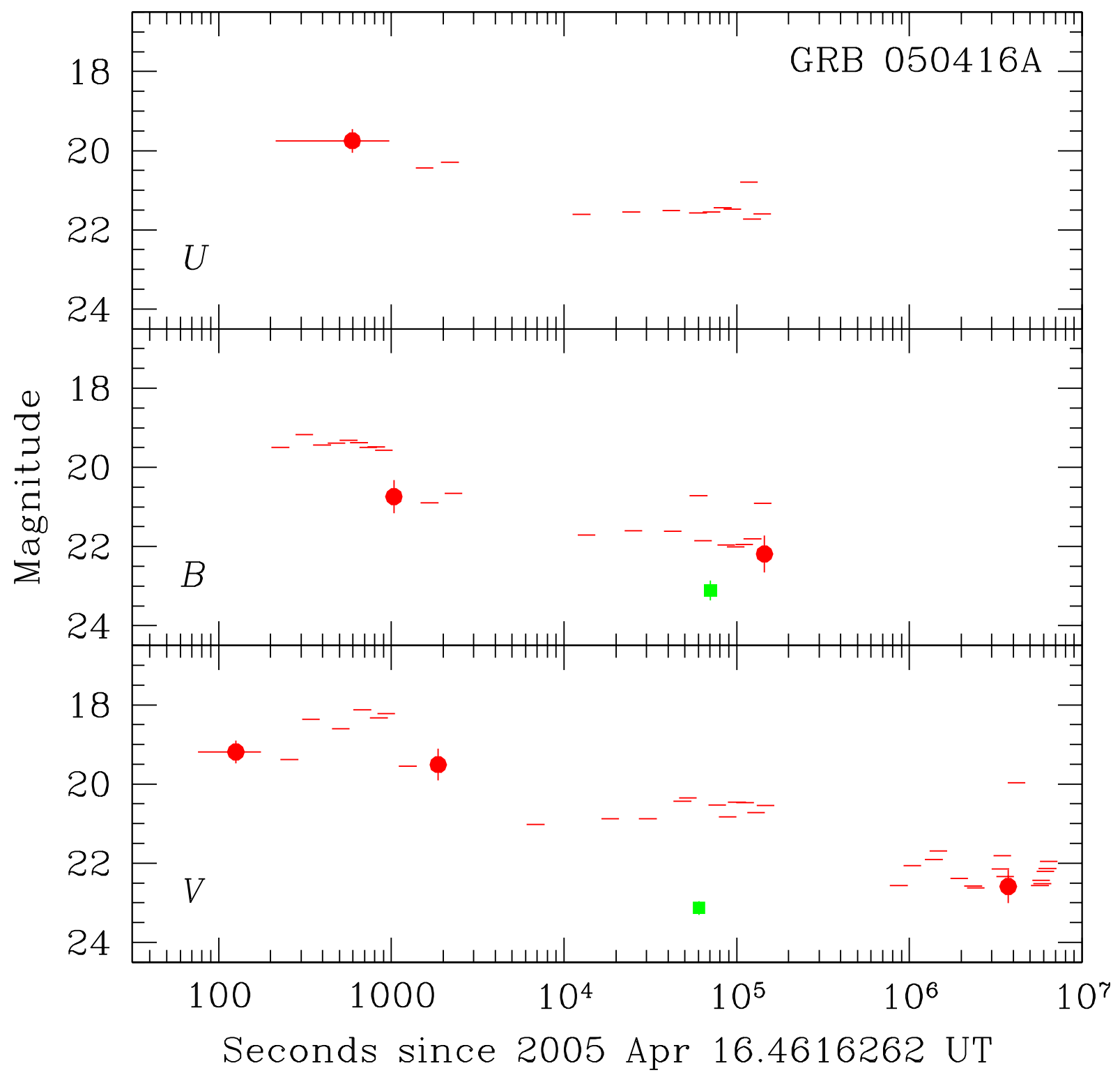

Fig. 3.- This Figure shows the UVOT (red, circles) and DFOSC (green, squares) $U$, $B$, and $V$ photometry of the afterglow of XRF 050416A. The details are the same as for Figure 2. 


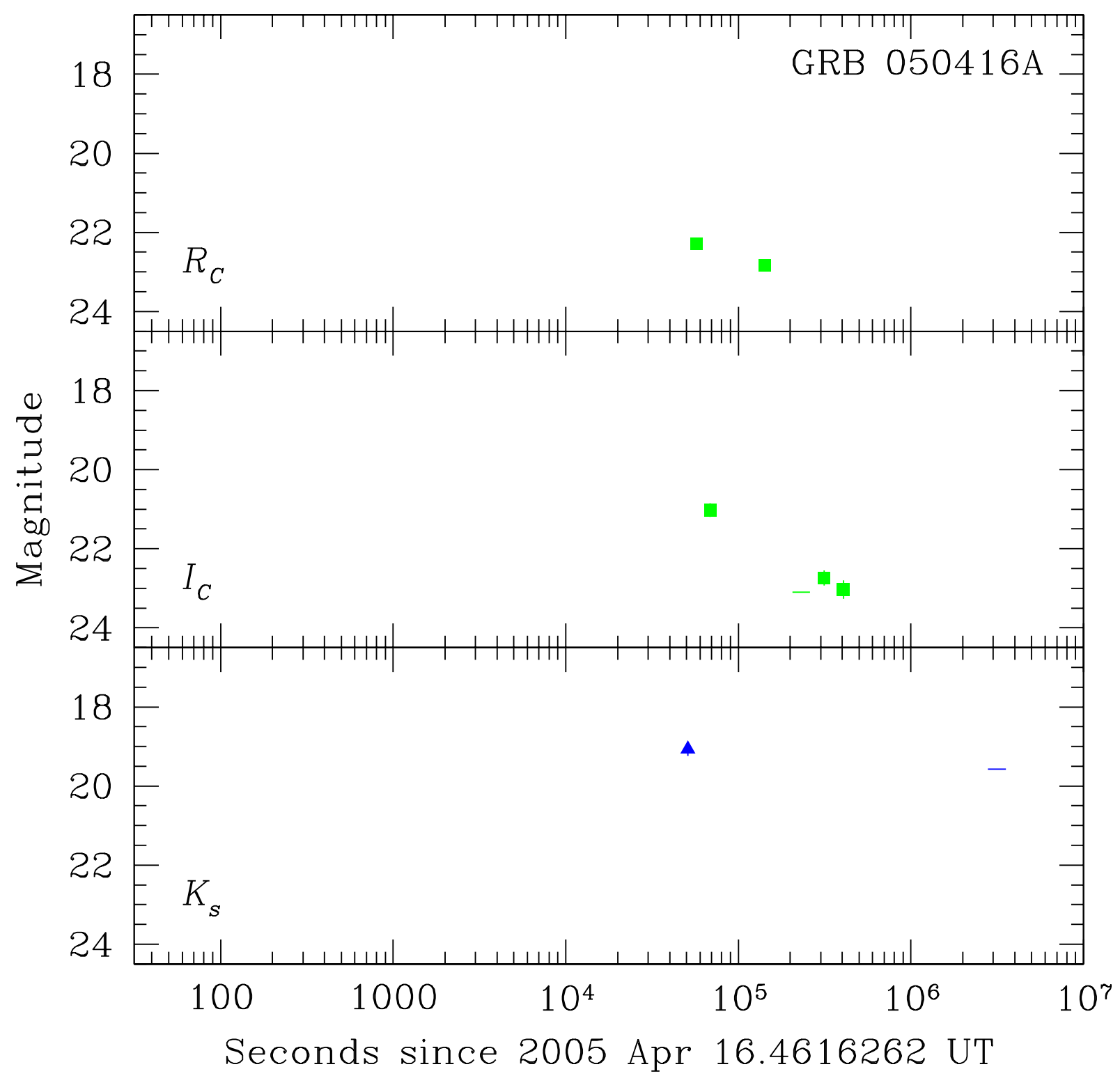

Fig. 4.- This Figure shows the DFOSC (green, squares) and NOTCam (blue, triangles) $R_{C}, I_{C}$, and $K_{s}$ photometry of the afterglow of XRF 050416A. The details are the same as for Figure 2. 
Table 1. Photometry of XRF 050416A. The time $t_{\text {mid }}$ is the time from the BAT trigger to the middle of the observation. The exposure time is denoted by $\Delta t$. Upper limits are $3 \sigma$ upper limits.

Errors are $1 \sigma$ statistical errors and do not include the systematic errors in the photometric zero points.

\begin{tabular}{|c|c|c|c|c|c|c|c|c|c|c|c|}
\hline$t_{\text {mid }}(\mathrm{s})$ & $\Delta t(\mathrm{~s})$ & Filter & Mag & Err & Instrument & $t_{\text {mid }}(\mathrm{s})$ & $\Delta t(\mathrm{~s})$ & Filter & Mag & Err & Instrument \\
\hline 126 & 100 & $V$ & 19.19 & 0.29 & UVOT & 59659 & 900 & $U$ & $>21.57$ & $\cdots$ & UVOT \\
\hline 228 & 10 & $B$ & $>19.49$ & $\ldots$ & UVOT & 60166 & 99 & $B$ & $>20.72$ & $\ldots$ & UVOT \\
\hline 257 & 10 & $V$ & $>19.39$ & $\ldots$ & UVOT & 60415 & $2 \times 1200$ & $V$ & 23.13 & 0.17 & DFOSC $^{\mathrm{a}}$ \\
\hline 285 & 10 & UVW1 & $>18.36$ & $\cdots$ & UVOT & 63859 & 900 & B & $>21.86$ & $\cdots$ & UVOT \\
\hline 313 & 10 & B & $>19.17$ & $\ldots$ & UVOT & 67559 & 9202 & UVM2 & 23.38 & 0.42 & UVOT $^{\mathrm{a}}$ \\
\hline 342 & 10 & $V$ & $>18.36$ & $\ldots$ & UVOT & 68693 & $3 \times 600$ & $I_{C}$ & 21.02 & 0.17 & DFOSC $^{\mathrm{a}}$ \\
\hline 355 & 10 & UVM2 & $>18.57$ & $\ldots$ & UVOT & 70541 & 900 & UVW1 & $>21.98$ & $\ldots$ & UVOT \\
\hline 369 & 10 & UVW1 & $>18.61$ & $\ldots$ & UVOT & 70549 & 1500 & B & 23.11 & 0.25 & DFOSC \\
\hline 397 & 10 & B & $>19.44$ & $\cdots$ & UVOT & 71393 & 791 & $U$ & $>21.55$ & $\ldots$ & UVOT \\
\hline 482 & 10 & B & $>19.39$ & $\ldots$ & UVOT & 73345 & 10146 & UVW2 & 23.25 & 0.33 & UVOT $^{\mathrm{a}}$ \\
\hline 497 & 10 & UVW2 & $>18.41$ & $\cdots$ & UVOT & 76988 & 459 & V & $>20.53$ & $\cdots$ & UVOT \\
\hline 510 & 10 & V & $>18.61$ & $\ldots$ & UVOT & 82070 & 900 & UVW1 & $>21.92$ & $\ldots$ & UVOT \\
\hline 566 & 10 & $B$ & $>19.32$ & $\ldots$ & UVOT & 82944 & 835 & $U$ & $>21.44$ & $\ldots$ & UVOT \\
\hline 594 & $10 \times 10$ & $U$ & 19.75 & 0.30 & UVOT $^{\mathrm{a}}$ & 86973 & 900 & $B$ & $>21.96$ & $\ldots$ & UVOT \\
\hline 650 & 10 & B & $>19.38$ & $\ldots$ & UVOT & 88743 & 810 & V & $>20.83$ & $\ldots$ & UVOT \\
\hline 666 & 10 & UVW2 & $>18.20$ & $\ldots$ & UVOT & 93637 & 900 & UVW1 & $>22.15$ & $\ldots$ & UVOT \\
\hline 679 & 10 & V & $>18.13$ & $\ldots$ & UVOT & 94514 & 841 & $U$ & $>21.47$ & $\ldots$ & UVOT \\
\hline 707 & 10 & UVW1 & $>19.30$ & $\ldots$ & UVOT & 98641 & 900 & $B$ & $>22.01$ & $\ldots$ & UVOT \\
\hline 735 & 10 & B & $>19.49$ & $\ldots$ & UVOT & 100162 & 311 & V & $>20.45$ & $\ldots$ & UVOT \\
\hline 792 & 10 & UVW1 & $>18.16$ & $\ldots$ & UVOT & 105335 & 900 & UVW1 & $>21.96$ & $\ldots$ & UVOT \\
\hline 819 & 10 & B & $>19.48$ & $\ldots$ & UVOT & 110415 & 900 & B & $>21.95$ & $\ldots$ & UVOT \\
\hline 848 & 10 & V & $>18.33$ & $\ldots$ & UVOT & 112038 & 514 & V & $>20.47$ & $\ldots$ & UVOT \\
\hline 876 & 10 & UVW1 & $>18.09$ & $\ldots$ & UVOT & 117400 & 900 & UVW1 & $>22.04$ & $\ldots$ & UVOT \\
\hline 904 & 10 & B & $>19.57$ & $\cdots$ & UVOT & 117970 & 223 & $U$ & $>20.80$ & $\ldots$ & UVOT \\
\hline 919 & 10 & UVW2 & $>19.05$ & $\ldots$ & UVOT & 122543 & 900 & $U$ & $>21.73$ & $\ldots$ & UVOT \\
\hline 933 & 10 & V & $>18.22$ & $\cdots$ & UVOT & 123434 & 868 & $B$ & $>21.80$ & $\ldots$ & UVOT \\
\hline 1035 & 100 & B & 20.74 & 0.42 & UVOT & 129343 & 624 & V & $>20.72$ & $\ldots$ & UVOT \\
\hline 1140 & 100 & UVW2 & $>19.81$ & $\ldots$ & UVOT & 135246 & 390 & UVW1 & $>21.34$ & $\ldots$ & UVOT \\
\hline 1245 & 100 & V & $>19.55$ & $\ldots$ & UVOT & 138805 & 401 & UVW1 & $>21.45$ & $\ldots$ & UVOT \\
\hline 1349 & 100 & UVM2 & $>20.75$ & $\ldots$ & UVOT & 140587 & 900 & $U$ & $>21.60$ & $\ldots$ & UVOT \\
\hline 1454 & 100 & UVW1 & $>20.32$ & $\ldots$ & UVOT & 141137 & 184 & $B$ & $>20.91$ & $\ldots$ & UVOT \\
\hline 1558 & 100 & $U$ & $>20.44$ & $\ldots$ & UVOT & 142008 & $25 \times 400$ & $R_{C}$ & 22.84 & 0.11 & DFOSC $^{a}$ \\
\hline 1663 & 100 & B & $>20.90$ & $\cdots$ & UVOT & 144815 & 900 & $B$ & 22.19 & 0.47 & UVOT \\
\hline 1768 & 100 & UVW2 & $>19.55$ & $\ldots$ & UVOT & 146738 & 553 & $V$ & $>20.55$ & $\ldots$ & UVOT \\
\hline 1868 & 93 & V & 19.51 & 0.40 & UVOT & 231672 & $53 \times 300$ & $I_{C}$ & $>23.10$ & $\ldots$ & DFOSC $^{a}$ \\
\hline 2081 & 100 & UVW1 & $>20.43$ & $\ldots$ & UVOT & 313726 & $29 \times 300$ & $I_{C}$ & 22.74 & 0.19 & DFOSC $^{\mathrm{a}}$ \\
\hline 2185 & 100 & $U$ & $>20.30$ & $\ldots$ & UVOT & 406438 & $21 \times 200$ & $I_{C}$ & 23.03 & 0.23 & DFOSC $^{a}$ \\
\hline 2290 & 100 & $B$ & $>20.65$ & $\cdots$ & UVOT & 868505 & 20957 & $V$ & $>22.56$ & $\ldots$ & UVOT $^{\mathrm{a}}$ \\
\hline 6850 & 900 & V & $>21.02$ & $\ldots$ & UVOT & 1039340 & 8274 & $V$ & $>22.06$ & $\ldots$ & UVOT $^{\mathrm{a}}$ \\
\hline 11730 & 900 & UVW1 & $>21.81$ & $\ldots$ & UVOT & 1384870 & 6755 & $V$ & $>21.91$ & $\ldots$ & UVOT $^{\mathrm{a}}$ \\
\hline 12637 & 900 & $U$ & $>21.61$ & $\ldots$ & UVOT & 1468840 & 4722 & $V$ & $>21.69$ & $\ldots$ & UVOT $^{\mathrm{a}}$ \\
\hline 13508 & 826 & $B$ & $>21.71$ & $\ldots$ & UVOT & 1941288 & 14167 & $V$ & $>22.38$ & $\cdots$ & UVOT $^{\mathrm{a}}$ \\
\hline 18532 & 900 & V & $>20.88$ & $\ldots$ & UVOT & 2338167 & 21133 & $V$ & $>22.58$ & $\ldots$ & UVOT $^{\mathrm{a}}$ \\
\hline 23601 & 900 & UVW1 & $>21.93$ & $\ldots$ & UVOT & 2424904 & 26389 & $V$ & $>22.63$ & $\ldots$ & UVOT $^{\mathrm{a}}$ \\
\hline 24508 & 900 & $U$ & $>21.55$ & $\ldots$ & UVOT & 3146746 & $39 \times 52$ & $K_{s}$ & $>19.57$ & $\ldots$ & NOTCam $^{a}$ \\
\hline 25231 & 530 & $B$ & $>21.60$ & $\ldots$ & UVOT & 3371121 & 12866 & $V$ & $>22.15$ & $\ldots$ & $\mathrm{UVOT}^{\mathrm{a}}$ \\
\hline
\end{tabular}


Table 1-Continued

\begin{tabular}{|c|c|c|c|c|c|c|c|c|c|c|c|}
\hline$t_{\text {mid }}(\mathrm{s})$ & $\Delta t(\mathrm{~s})$ & Filter & Mag & Err & Instrument & $t_{\text {mid }}(\mathrm{s})$ & $\Delta t(\mathrm{~s})$ & Filter & Mag & Err & Instrument \\
\hline 30599 & 900 & $V$ & $>20.88$ & $\ldots$ & UVOT & 3455561 & 4722 & $V$ & $>21.81$ & $\ldots$ & UVOT $^{\mathrm{a}}$ \\
\hline 36640 & 858 & UVW1 & $>22.00$ & $\ldots$ & UVOT & 3590733 & 15986 & $V$ & $>22.34$ & $\ldots$ & UVOT $^{\mathrm{a}}$ \\
\hline 41791 & 900 & $U$ & $>21.51$ & $\cdots$ & UVOT & 3732868 & 18806 & $V$ & 22.59 & 0.42 & UVOT $^{\mathrm{a}}$ \\
\hline 42552 & 606 & $B$ & $>21.61$ & $\ldots$ & UVOT & 4170209 & 321 & $V$ & $>19.97$ & $\ldots$ & UVOT $^{\mathrm{a}}$ \\
\hline 48456 & 373 & $V$ & $>20.44$ & $\cdots$ & UVOT & 5706808 & 25285 & $V$ & $>22.57$ & $\cdots$ & UVOT $^{\mathrm{a}}$ \\
\hline 51023 & $36 \times 78$ & $K_{s}$ & 19.07 & 0.17 & NOTCam $^{\mathrm{a}}$ & 5793106 & 20898 & $V$ & $>22.43$ & $\ldots$ & UVOT $^{\mathrm{a}}$ \\
\hline 52031 & 271 & $V$ & $>20.35$ & $\ldots$ & UVOT & 5880015 & 23533 & $V$ & $>22.52$ & $\ldots$ & UVOT $^{\mathrm{a}}$ \\
\hline 54363 & 132 & UVW1 & $>20.78$ & $\ldots$ & UVOT & 6136666 & 15285 & $V$ & $>22.21$ & $\ldots$ & UVOT $^{\mathrm{a}}$ \\
\hline 57086 & $6 \times 900$ & $R_{C}$ & 22.29 & 0.08 & DFOSC $^{a}$ & 6310565 & 13933 & $V$ & $>22.13$ & $\ldots$ & UVOT $^{\mathrm{a}}$ \\
\hline 57990 & 773 & UVW1 & $>21.70$ & $\ldots$ & UVOT & 6394649 & 10004 & $V$ & $>21.95$ & $\ldots$ & UVOT $^{\mathrm{a}}$ \\
\hline
\end{tabular}

${ }^{\mathrm{a}}$ This data point consists of coadded data. 
We performed photometry on each UVOT exposure using a circular aperture with a radius of $2^{\prime \prime}$ centred on the position of the optical afterglow. This radius is approximately equal to the full-width at half-maximum (FWHM) of the UVOT point-spread function (PSF). The PSF varies with filter and with the temperature of the telescope, so we did not match the extraction aperture to the PSF for each exposure. The PSF FWHM, averaged over the temperature variations, ranges from $1^{\prime \prime} .79 \pm 0$ ' 05 for the $V$ filter to 2 .' $17 \pm 0$ ' 03 for the UVW2 filter. The background was measured in a sky annulus of inner radius $17^{\prime \prime} .5$ and width $5^{\prime \prime}$ centred on the afterglow. The source was not detected in the individual ultraviolet filter exposures, so these exposures were coadded. The source was detected in the coadded UVW2 and UVM2 exposures, but not in the coadded UVW1 exposure.

Aperture corrections were computed for each exposure to convert the $2^{\prime \prime}$ photometry to the standard aperture radii used to define UVOT's photometric zero points ( $6^{\prime \prime}$ for $U B V$ and $12^{\prime \prime}$ for the ultraviolet filters). Six isolated stars were used to compute the aperture correction for each exposure. The RMS scatter in the mean aperture correction for a single exposure was typically $\approx 0.02$ mag. The RMS scatter for each exposure was added in quadrature to the statistical error in the $2^{\prime \prime}$ magnitude to obtain the total $1 \sigma$ error in each point. All detections above the $2 \sigma$ significance level are tabulated in Table 1.

The UVOT is a photon-counting device with a frame read-out time of $11.0329 \mathrm{~ms}$. It is only able to record one photon per detector cell during each read out. This results in coincidence losses at high count rates. For very high count rates, corresponding to $V \lesssim 13.5$, these losses are significant and can dramatically affect the photometry, so coincidence loss corrections must be made. We have corrected all of our data for coincidence loss, however the afterglow has $V>19$, so coincidence losses are negligible, typically less than $0.01 \mathrm{mag}$, for the afterglow. Coincidence loss corrections, however, are significant for the stars used to compute the aperture corrections.

The zero points used to transform the instrumental UVOT magnitudes to Vega magnitudes were taken from in-orbit measurements as obtained from the HEASARC Swift/UVOT Calibration

Database (CalDB) ${ }^{2}$ dated 2005-11-18 in the file swuphoт20041120v102.FITs. Colour terms were not applied to the photometric calibrations, but preliminary calibrations of on-orbit data suggest that they are negligible.

\subsection{Ground-Based Data}

\subsection{1. $D F O S C$}

The XRF 050416A optical afterglow was observed with the $1.54 \mathrm{~m}$ Danish telescope equipped with DFOSC. DFOSC is focal reducer camera based on a backside illuminated EEV/MAT CCD, providing a pixel scale of $0{ }^{\prime} \cdot 39$ per pixel and a field of view of $13.6 \times 13.6$. The DFOSC observations

\footnotetext{
${ }^{2}$ http://swift.gsfc.nasa.gov/docs/heasarc/caldb/swift/
} 
were carried out on five consecutive nights starting on 2005 Apr 17.1 UTC (see Table 1 for further details). Data reduction was performed following standard procedures (bias level subtraction and sky flat fielding) running under IRAF ${ }^{3}$.

Photometry was performed on the combined DFOSC images using the SExTRACTOR software (Bertin \& Arnouts 1996) with its default parameters. Magnitudes and magnitude errors were determined using the MAG_AUTO option. The DFOSC data were calibrated using the $B V R_{C} I_{C}$ field photometry of Henden (2005). All of the stars that were in common between the Henden (2005) photometry and our SExTRACTOR photometry were matched and used to compute photometric zero points for each DFOSC image. Colour terms were not used as they did not improve the quality of the calibration.

\subsubsection{NOTCam}

Near-infrared observations of XRF 050416A were taken on 2005 Apr 17 and 2005 May 22 with NOTCam mounted on the Nordic Optical Telescope. NOTCam is a multi-mode instrument that is based on a $1024 \times 1024 \mathrm{HgCdTe}$ "HAWAII" detector providing a scale of $0^{\prime \prime} 233$ per pixel and a field of view of $4^{\prime} \times 4^{\prime}$. Data reduction was done by following a standard procedure for near-infrared imaging (shift and expose, subtract a scaled median sky, divide by twilight flat, align and combine by adaptive sigma clipping). The individual images were aligned from the centroid of the star $12335122+2104142$ in the 2MASS catalogue (Skrutskie et al. 2006).

Photometry was performed on the NOT images using SExTRACTOR with the default parameters in the same way as for the DFOSC images. The NOTCam photometry was calibrated using star $12335122+2104142$. The 2MASS catalogue magnitude of this star is $K_{s}=11.383 \pm 0.018$ (Skrutskie et al. 2006). Our infrared photometry is listed in Table 1.

\section{Results}

\subsection{The Decay Rate}

We use our photometry to constrain the rate of a power law decay of the afterglow in each filter where there is multi-epoch photometry. The $X$-ray data (Mangano et al. 2006) shows a change in the $X$-ray light curve at 1450 seconds which Mangano et al. (2006) interpret as the end of energy injection. In order to directly compare the ultraviolet/optical/infrared decay rate to the $X$-ray decay rate we only consider photometry with mid-point times more than $1450 \mathrm{~s}$ after the BAT trigger. This is the period that Mangano et al. (2006) call Phase C and corresponds to the time

\footnotetext{
${ }^{3}$ IRAF is distributed by the National Optical Astronomy Observatories, which are operated by the Association of Universities with National Science Foundation.
} 
after the second $X$-ray break.

We find $\alpha_{B}=+1.18 \pm 0.68, \alpha_{V}=-0.96 \pm 0.53, \alpha_{R}=-0.57 \pm 0.14$, and $\alpha_{I}=-1.04 \pm 0.66$. It is not clear if the increase in the $B$-band flux at $144815 \mathrm{~s}(\approx 1.7$ days $)$ is real or a noise spike causing the source to appear brighter than it really is. The mean decay rate, excluding the $B$-band data, is $\bar{\alpha}=-0.86 \pm 0.15$ (standard error). This is in good agreement with the $X$-ray decay rate of $\alpha_{X}=-0.88 \pm 0.02$ at the same time. Further, the decay rates in each filter, excluding $B$, are all within $2 \sigma$ of the $X$-ray value. This suggests that, for $t>1450 \mathrm{~s}$, there is no spectral break between the $X$-ray and optical bands. As a further test we used the spectral energy distribution from Section 3.2 to convert all the photometry to the $R_{C}$ band and fit a single power law. This yielded a slope of $-0.75 \pm 0.19$, in good agreement with the mean slope that we determined above. We prefer to adopt the mean slope since it does not depend on uncertainties, and possible temporal variations, in the spectral shape.

The early time UVOT $V$-band photometry is consistent with the afterglow having a constant $V$-band magnitude between 126 and 1868 seconds after the BAT trigger. The decay rate in this interval is $\alpha_{V}=-0.11 \pm 0.17$. This is consistent with the $X$-ray slope during Phase B $(-0.44 \pm 0.13)$ (Mangano et al. 2006) at the $1.6 \sigma$ level.

\subsection{The Spectral Energy Distribution}

We constructed the spectral energy distribution between $1930 \AA$ and $22200 \AA$ at 60415 s after the BAT trigger. The photometry data nearest this time for each filter were transformed to 60415 s assuming a decay rate of $\alpha=-0.88$. We adopted the $X$-ray decay rate instead of the optical decay rate that we determined in Section 3.1 because the $X$-ray decay is better constrained, and we believe that the $X$-ray and optical decays are the same (see Sect. 3.1). The UVW2 and UVM2 magnitudes were transformed to flux densities using the conversion factors in the Swift/UVOT CalDB. The optical and infrared magnitudes were converted to flux densities using the zero points of Fukugita et al. (1995) and Cox (2000). Each data point was corrected for Galactic extinction using the reddening value of Schlegel et al. (1998) (see Section 2) but not for any extinction that may be present in the host galaxy or in intergalactic space along the line of sight to the burst. The SED is shown in Figure 5.

The SED was fit by $f_{\nu}(\nu) \propto \nu^{\beta} 10^{-0.4 A(\nu)}$ where $f_{\nu}(\nu)$ is the flux density at frequency $\nu, \beta$ is the intrinsic spectral index, and $A(\nu)$ is the extragalactic extinction in the host $(z=0.6535)$ at frequency $\nu$. We found that the best fit occurred for the Small Magellanic Cloud (SMC) extinction law of Pei (1992), although using a Milky Way or Large Magellanic Cloud extinction law does not significantly change the results. We prefer the SMC since it provides good fits to the extinction seen

in the host galaxies of other GRBs (e.g., Holland et al. 2003). If we fix the intrinsic spectral slope to the $X$-ray value of $\beta_{X}=-1.04$ (Mangano et al. 2006) the extinction in the host is $A_{V}=0.24 \pm 0.06$ mag. If we allow both $\beta$ and $A_{V}$ to be free parameters the best fit occurs for $\beta=-1.14 \pm 0.20$ and 


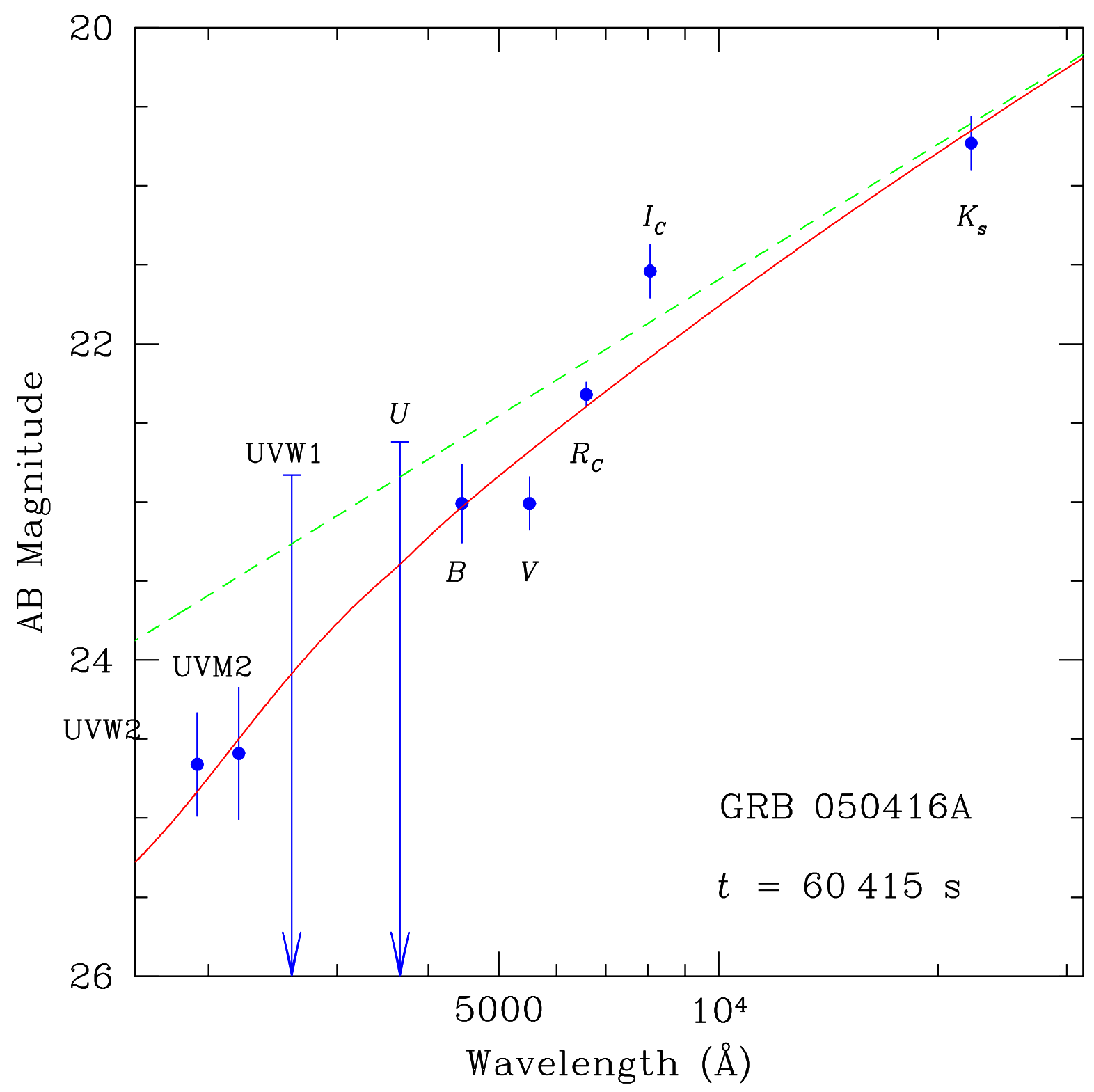

Fig. 5. - This Figure shows the SED of the ultraviolet/optical/infrared afterglow of XRF 050416A at $60415 \mathrm{~s}$ (0.7 days) after the BAT trigger. The filled circles represent observed photometry corrected for extinction in the Milky Way. The solid line represents the SED fit with an extinction in the host of $A_{V}=0.19 \pm 0.11 \mathrm{mag}$ assuming an intrinsic power law spectrum with a slope of $\beta=-1.14 \pm 0.20$. The dashed line shows the unreddened spectrum. 
$A_{V}=0.19 \pm 0.11$. Therefore, we believe that there is approximately 0.2 mag of $V$-band extinction in the host along the line of sight to XRF 050416A.

The low value of the extinction in the host is at odds with the high neutral hydrogen column density found by fitting the $X$-ray spectrum (Mangano et al. 2006). Predehl \& Schmitt (1995) find $N_{\mathrm{H}}=\left(1.79 \times 10^{21}\right) A_{V}$ for the the conversion between hydrogen column density and extinction in the Milky Way. Using the value of $N_{\mathrm{H}}$ derived from the $S$ wift/XRT $X$-ray observations (Mangano et al. 2006) we find $A_{V}=3.8_{-0.7}^{+0.6}$, which implies significant extinction in the host along the line of sight to XRF 050416A. The discrepancy between this and the small amount of extinction that we find from the ultraviolet/optical/infrared SED suggests that the relationship between hydrogen gas and dust is different in the host of XRF 050416A than it is in the Milky Way. Combining the $X$-ray hydrogen column density with our estimate of $A_{V}$ gives $N_{\mathrm{H}}=\left(36_{-22}^{+21} \times 10^{21}\right) A_{V}$ in the host galaxy. This is consistent with the $N_{\mathrm{H}} / A_{V}$ relation found in the $\mathrm{SMC}$ of $N_{\mathrm{H}}=\left(15.4 \times 10^{21}\right) A_{V}$ using Eq. 4 and Table 2 of Pei (1992).

The high gas-to-dust ratio seen in the host of XRF 050416A is typical of other GRB host galaxies, such as GRB 000301C (Jensen et al. 2001), GRB 000926 (Fynbo et al. 2001), and GRB 020124 (Hjorth et al. 2003), all of which have ratios consistent with that observed in the SMC, (see also Stratta et al. 2004; Kann et al. 2006). This suggests that our choice of using an SMC extinction law to determine the intrinsic spectral slope is reasonable. The high gas-to-dust ratio may be a consequence of dust destruction by the ultraviolet and $X$-ray flux from the GRB as described by (e.g. Waxman \& Draine 2000; Perna et al. 2003). Alternately, it may indicate that the star formation in the host is fairly recent and that there may not have been time for large amounts of dust to form.

\subsection{Cooling Break}

The $V R_{C} I_{C}$ photometry yields a mean decay index of $\alpha=-0.86 \pm 0.15$ for times more than 1450 seconds after the burst. The ultraviolet/optical/infrared SED at $60415 \mathrm{~s}$ has a slope of $\beta=-1.14 \pm 0.20$, after correcting for extinction in the host galaxy. This is consistent with the intrinsic spectral slope derived from the $X$-ray data. The lack of evidence for a change in either the intrinsic spectral slope or the decay rate between the $X$-ray and optical bands suggests that there is no spectral break between these two regimes. Therefore the synchrotron cooling frequency must lie either above the $X$-ray band or below the $K_{s}$ band at $60415 \mathrm{~s}(\approx 0.7$ days $)$ after the BAT trigger.

The spectral and temporal decay rates can be used to predict the index of the electron energy distribution, $p$. The decay rate will be a more robust number than the spectral index since the spectral slope may be affected by uncertainties in the absorption along the line of sight. After the jet break the magnitude of the decay index should equal the value of the electron index. However, electron indices of less than unity are unphysical. Therefore, we can rule out a jet break before 42 
days after the BAT trigger because the $X$-ray decay rate remains constant at -0.88 between 1450 $\mathrm{s}$ and at least 42 days.

In order to determine the location of the cooling break, and to estimate the value of the electron index, we use closure relations between $\alpha$ and $\beta$. These are based on the relations of Dai \& Cheng (2001) when $1<p<2$. For $p \geq 2$ the relations of Sari et al. (1999) were used for the case of a homogeneous circumburst environment, and the relations of Chevalier \& Li (1999) were used for the case of a pre-existing stellar wind. Table 2 lists the closure relations and their observed values. None of the closure relations for $p \geq 2$ give values consistent with zero. Therefore, we believe that $1<p<2$. This is a low value for a GRB, but three of the ten bursts studied by Panaitescu \& Kumar (2002) had $p \approx 1.4$, so it can not be ruled out. For $1<p<2$ the case of a homogeneous circumburst medium with $\nu_{X}<\nu_{c}$, and the cases of a pre-existing stellar wind with $\nu_{X}<\nu_{c}$ or $\nu_{c}<\nu_{\mathrm{opt}}$ all give comparable closure values that are consistent with zero.

If $1<p<2$ then the predicted intrinsic spectral slope is between 0 and -0.5 if the cooling break is above the $X$-ray band and between -0.5 and -1 if the cooling break is below the optical bands. Both the $X$-ray and ultraviolet/optical/infrared spectral slopes are consistent with $\beta=-1$, which corresponds to $p=3$ for $\nu_{X}<\nu_{c}$, which is unusually high for a GRB. However, if $\nu_{c}<\nu_{\mathrm{opt}}$ then $p=2$, which is fairly typical for GRBs. An electron index of $p=2$ is consistent with cases 5 and 7 of the closure relations in Table 2. In these two cases the predicted optical decay is $\alpha=-1$ regardless of the density structure of the environment that the burst is expanding into. This decay slope is slightly steeper than the observed decay rate of $-0.86 \pm 0.15$, but it is within the observed uncertainties. Therefore, we believe that $p \approx 2$ and $\nu_{c}<\nu_{\text {opt }}$ for XRF 050416A at $60415 \mathrm{~s}$ after the BAT trigger.

\subsection{Energy Considerations}

Sakamoto et al. (2006) find that the isotropic equivalent energy of XRF 050416A is $1.2 \times 10^{51}$

Table 2. This Table lists the closure relationships for the various cases under consideration. The theoretical value of each closure relation is zero.

\begin{tabular}{cclccc}
\hline \hline Case & Model & Environment & $p$ & \multicolumn{1}{c}{ Closure } & Value \\
\hline 1 & $\nu_{\mathrm{opt}}<\nu_{X}<\nu_{c}$ & ISM & $1<p \leq 2$ & $\alpha-3 / 8 \beta+9 / 16$ & $+0.13 \pm 0.19$ \\
2 & & & $p>2$ & $\alpha-3 / 2 \beta$ & $+0.85 \pm 0.11$ \\
3 & & Wind & $1<p \leq 2$ & $\alpha-1 / 4 \beta+9 / 8$ & $+0.55 \pm 0.16$ \\
4 & & & $p>2$ & $\alpha-3 / 2 \beta+1 / 2$ & $+0.35 \pm 0.11$ \\
5 & $\nu_{c}<\nu_{\mathrm{opt}}<\nu_{X}$ & ISM & $1<p \leq 2$ & $\alpha-3 / 8 \beta+5 / 8$ & $+0.19 \pm 0.19$ \\
6 & & & $p>2$ & $\alpha-3 / 2 \beta-1 / 2$ & $+0.55 \pm 0.11$ \\
7 & & Wind & $1<p \leq 2$ & $\alpha-1 / 4 \beta+3 / 4$ & $+0.18 \pm 0.16$ \\
8 & & & $p>2$ & $\alpha-3 / 2 \beta-1 / 2$ & $+0.55 \pm 0.11$ \\
\hline
\end{tabular}


erg. This, and the observed limits on the jet break time, can be used to estimate the opening angle of the jet and thus the total gamma-ray energy of the burst (Rhoads 1999; Sari et al. 1999; Frail et al. 2001). The jet opening angle for XRF 050416A is

$$
\theta_{j}=0.106 t_{j}^{3 / 8}\left(\eta_{\gamma} / 0.2\right)^{1 / 8}(n / 0.1)^{1 / 8}
$$

where $t_{j}$ is the observed jet break time in days since the BAT trigger, $\eta_{\gamma}$ is the efficiency of converting energy in the ejecta into gamma rays, and $n$ is the particle density in $\mathrm{cm}^{-3}$. The $X$-ray light curve (Mangano et al. 2006) suggests that there is no jet break at times earlier than 42 days. Setting $t_{j} \geq 42$ yields $\theta_{j} \geq 25^{\circ}$ assuming $\eta_{\gamma}=0.2$ and $n=0.1 \mathrm{~cm}^{-3}$. This result is not strongly sensitive to our choices of $\eta_{\gamma}$ and $n$. The corresponding energy in gamma rays, corrected for beaming, is $E_{\gamma} \geq 1.1 \times 10^{50} \mathrm{erg}$. The upper limit on the gamma-ray energy can be obtained from the case where there is no beaming (and thus no jet break). In that case $E_{\gamma}=E_{\text {iso }}$, which implies that $1.1 \times 10^{50} \mathrm{erg} \leq E_{\gamma} \leq 1.2 \times 10^{51} \mathrm{erg}$. Therefore the total energy must be less than the canonical GRB energy of Bloom et al. (2003) if there is any beaming.

Sakamoto et al. (2006) showed that XRF 050416A fits onto the Amati relation (Amati et al. 2002) between $E_{\text {iso }}$ and $E_{p}^{\prime}$. They also showed that this burst can not be made to satisfy the Ghirlanda relation (Ghirlanda et al. 2004) between $E_{\gamma}$ and $E_{p}^{\prime}$ due to the lack of a jet break out to 35 days after the BAT trigger. Mangano et al. (2006) have shown that there is no jet break to at least 42 days, which makes the discrepancy with the Ghirlanda relation even larger.

The Liang \& Zhang (2005) relation

$$
\frac{E_{\text {iso }}}{10^{53} \mathrm{erg}}=0.85 \times\left(\frac{E_{p}^{\prime}}{100 \mathrm{keV}}\right)^{1.94} \times\left(\frac{t_{j}^{\prime}}{1 \text { day }}\right)^{-1.24}
$$

implies that the jet break should occur at $\approx 1.5$ days after the BAT trigger in the observer's frame. If we assume that XRF $050416 \mathrm{~A}$ has a two-component jet with a narrow component that breaks at 1.5 days the observed decay between 1.5 and 42 days will be the sum of the post-narrow-jet break component for the narrow jet and the post-cooling break component for the wide jet. A two-component jet model has been proposed to explain some of the observed features in the decays of GRB 030329 (Berger et al. 2003; Sheth et al. 2003), such as the presence of two achromatic breaks. We propose that the observed optical afterglow of XRF 050416A may be the sum of two jets that both conform with the Ghirlanda relation and the Liang \& Zhang relation. In this picture the narrow jet would have an opening angle of $\theta_{j, n} \approx 7^{\circ}$ assuming $t_{j, n}=1.5$ days, and the wide jet would have $\theta_{j, w} \gtrsim 25^{\circ}$ for a wide jet break time of $t_{j, w}>42$ days. The gamma-ray energies in each jet are $E_{\gamma, n} \approx 5 \times 10^{48} \mathrm{erg}$ and $E_{\gamma, w} \gtrsim 7 \times 10^{49} \mathrm{erg}$, which is roughly consistent with the division of energy in the two-component jet model for GRB 030329.

Peng et al. (2005) find that for two component jets where the total energy in the wide jet is greater than that in the narrow jet the wide jet will dominate the optical afterglow after approx- 
imately $0.1-1$ days. Since the deceleration time of the wide jet is similar to the break time of the narrow jet the emergence of the wide jet at this time can mask the steepening of the light curve caused by the jet break in the narrow component. We suggest that this is what has happened in XRF 050416A. We see no evidence for a change in the spectral slope or the decay rate between the optical and $X$-ray regimes at $60415 \mathrm{~s}(\approx 0.7$ days $)$. This implies that both the narrow and wide components have cooling frequencies below the optical band at this time.

\subsection{Constraints on a Supernova Component}

UVOT observation in the $V$-band filter were taken up to 74 days after the BAT trigger. We searched the late time exposure for evidence of a rebrightening. There is one low-significance UVOT detection of the afterglow after $144815 \mathrm{~s}(1.676 \mathrm{~d})$. There is a second low-significance detection at $3732868 \mathrm{~s}$ (43 d). A visual examination of the second detection suggests that it is a noise spike in the data and not a point source.

A type Ib/c supernova like SN1998bw (Patat \& Piemonte 1998) at the distance of XRF 050416A is expected to peak at approximately $10(1+z) \approx 16$ days after the burst. We find no evidence for a source at the location of the afterglow at this time down to a $3 \sigma$ limiting magnitude of $V_{\lim }=21.9$ in coadded exposures. For $z=0.6535$ the observed $V$ band approximately corresponds to the rest frame $U$ band, so our upper limit corresponds to $M_{U}<-21.1$. SN1998bw had a peak $U$-band absolute magnitude of $M_{U}=-19.16$ (Galama et al. 1998) so the UVOT data is not able to constrain the existence of a supernova component to the afterglow of XRF 050416A.

\section{Conclusions}

XRF 050416A is a near-by $(z=0.6535)$, short $\left(T_{90}=2.4 \mathrm{~s}\right)$ XRF that shows no evidence for a jet break out to at least 42 days after the burst. The spectral slope and decay rate are the same in the optical as in Phase $\mathrm{C}$ of the $X$-ray decay (Mangano et al. 2006). This suggests that there is no cooling break between approximately $1.24 \AA(10 \mathrm{keV})$ and $22000 \AA(0.006 \mathrm{keV})$ at $60415 \mathrm{~s}$ after the burst. Further, the constancy of the decay suggests that the cooling break did not pass through the optical between $1450 \mathrm{~s}$ (0.016 days) and $406438 \mathrm{~s}$ (4.7 days), nor did it pass through the $X$-ray regime between $1450 \mathrm{~s}$ ( 0.016 days) and $\approx 3.6 \times 10^{6} \mathrm{~s}$ (42 days). We find the best agreement with the synchrotron model occurs if the cooling break is below the optical at $60415 \mathrm{~s}$ (0.7 days) after the burst.

The optical light decay slope is $\alpha=-0.86 \pm 0.15$ and the intrinsic ultraviolet/optical/infrared spectral slope is $\beta=-1.14 \pm 0.20$. The best estimate of the electron index is $p \approx 2$. We are unable to distinguish between a burst occurring in a homogeneous environment or a wind-stratified

one. The lack of a jet break out to $\gtrsim 42$ days implies that the jet opening angle is $>25^{\circ}$ and the gamma-ray energy is $1.1 \times 10^{50} \mathrm{erg} \leq E_{\gamma} \leq 1.2 \times 10^{51} \mathrm{erg}$. If XRF 050416A has the canonical 
energy of Bloom et al. (2003) then the burst is not beamed.

An alternate interpretation of the data is that XRF 050416A has a two component jet. The narrow component has an opening angle of $\theta_{j, n} \approx 7^{\circ}$ and experienced a jet break at 1.5 days, in

agreement with the prediction of the Liang \& Zhang (2005) relation between $E_{\text {iso }}, E_{p}^{\prime}$, and $t_{j}^{\prime}$. The wide component does not break until at least 42 days after the burst and must have an opening angle of $\gtrsim 25^{\circ}$. The wide component jet contains approximately 14 times more energy than the narrow component jet does. This behaviour is similar to what was seen in GRB 030329 (Berger et al. 2003; Sheth et al. 2003).

We find no evidence for a large amount of extinction along the line of sight to XRF 050416A. The best fit ultraviolet/optical/infrared spectrum suggests that $A_{V} \approx 0.2$ mag in the host galaxy. This is inconsistent with the large hydrogen column density implied by the $X$-ray spectrum. If we assume that the gas-to-dust ratio in the host is the same as that in the Milky Way the implied extinction is $A_{V}=3.8_{-0.7}^{+0.6} \mathrm{mag}$. Using the extinction derived from the optical data and the hydrogen column density derived from the $X$-ray data the gas-to-dust ratio in the host is $N_{\mathrm{H}} / A_{V}=$ $3.6_{-2.2}^{+2.1} \times 10^{22}$. This is dramatically larger than it is in the Milky Way, but consistent with what is seen in the SMC, and in the host galaxies of other GRBs.

The authors wish to thank Scott Barthelmy, and the GRB Coordinates Network (GCN) for rapidly providing precise GRB positions to the astronomical community. This research has made use of the NASA/IPAC Extragalactic Database (NED), which is operated by the Jet Propulsion Laboratory, California Institute of Technology, under contract with NASA. This publication makes use of data products from the Two Micron All Sky Survey, which is a joint project of the University of Massachusetts and the Infrared Processing and Analysis Center/California Institute of Technology, funded by the National Aeronautics and Space Administration and the National Science Foundation. We acknowledge the use of public data from the Swift data archive. This paper is based, in part, on observations taken with the Nordic Optical Telescope, operated on the island of Santa Miguel de la Palma jointly by Denmark, Finland, Iceland, Norway, and Sweden in the Spanish Observatorio del Roque de los Muchachos of the Instituto de Astrofísica de Canarias. STH would like to thank the DARK Cosmology Centre for its hospitality while writing part of this paper. The DARK Cosmology Centre is funded by the Danish National Research Foundation. This work is sponsored at Penn State University by NASA's office of Space Science through contract NAS5-00136 and at MSSL by funding from PPARC. The research of JG is supported by the Spanish Ministry of Science and Education through programmes ESP2002-04124-C03-01 and AYA2004-01515.

\section{REFERENCES}

Amati, L., Frontera1, F., Tavani, M., in't Zand, J. J. M., Antonelli, A., Costa, E., Feroci, M., Guidorzi, C., 2002, A\&A, 390, 81 
Anderson, G., Salvo, M., Rich, J., \& Schmidt, B. P., 2005, GCN Circular 3266

Barthelmy, S. D., Barbier, L. M., Cummings, J. R., Fenimore, E. E., Gehrels, N., Hullinger, D., Krimm, H. A., Markwardt, C. B., et al., 2005, Sp. Sci. Rev., 120, 143

Berger, E., Kulkarni, S. R., Pooley, G., Frail, D. A., McIntyre, V., Wark, R. M., Sari, R., Soderberg, A. M., et al., 2003, Nature, 126, 154

Bertin, E., \& Arnouts, S., 1996, A\&AS, 117, 393

Bloom, J. S., Frail, D. A., \& Kulkarni, S., 2003, ApJ, 594, 674

Burrows D. N., Hill, J. E., Nousek, J. A., Kennea, J. A., Wells, A., Osborne, J. P., Abbey, A. F., Beardmore, A., et al., 2005, Sp. Sci. Rev., 120, 165

Cenko, S. B., \& Fox, D. B., 2005, GCN Circular 3265

Cenko, S. B., Kulkarni, S. R., Gal-Yam. A., Berger, E., 2005, GCN Circular 3542

Chevalier, R. .A. \& Li, Z.-Y., 1999, ApJ, 520, L29

Cox, A. N., ed., 2000, Allen's Astrophysical Quantities (4th ed; New York: Springer)

Dai, Z. G., \& Cheng, K. S., 2001, ApJ, 558, L109

Fox, D. B., 2005, GCN Circular 3408

Frail, D. A., Kulkarni, S. R., Sari, R., Djorgovski, S. G., Bloom, J. S., Galama, T. J., Reichart, D. E., Berger, E., et al., 2001, ApJ, 562, L55

Fukugita, M., Shimasaku, K., \& Ichikawa, T., 1995, PASP, 107, 94

Fynbo, J. P. U., Gorosabel, J., Dall, T. H., Hjorth, J., Pedersen, H., Andersen, M. I.; Møller, P., Holland, S. T., et al., 2001, A\&A, 373, 796

Fynbo, J. P. U., Jensen, B. L., Hjorth, J., Wiersema, K., Starling, R., Vreswijk, P., Rol, E., Levan, A., et al., 2005b, GCN Circular 3176

Galama, T. J., Vreeswijk, P. M., van Paradijs, J., Kouveliotou, C., Augusteijn, T., Bohnhardt, H., Brewer, J. P.; Doublier, V., et al., 1998, Nature, 395, 670

Gehrels, N., Chincarini, G., Ciommi, P., Mason, K. O., Nousek, J. A., Wells, A. A., White, N. E., Barthelmey, S. D., et al., 2004, ApJ, 611, 1005

Ghirlanda, G., Ghisellini, G., \& Lazzati, D., 2004, ApJ, 616, 331

Henden, A., 2005, GCN Circular 3454 
Hjorth, J., Møller, P., Gorosabel, J., Fynbo, J. P. U., Toft, S., Jaunsen, A. O., Kaas, A. A., Purismo, T., Torii, K., et al., 2003, ApJ, 597, 699

Holland, S. T., Weidinger, M., Fynbo, J. P. U., Gorosabel, J., Hjorth, J., Pedersen, K., Méndez, A. J., Augusteijn, T., et al., 2003, AJ, 125, 2291

Jensen, B. L., Fynbo, J. P. U., Gorosabel, J., Hjorth, J., Holland, S. T., Møller, P., Thomsen, B., Björnsson, G., et al., 2001, A\&A, 370, 909

Kann, D. A., Klose, S., \& Zeh, A., 2006, ApJ, 641, 993

Kennea, J. A., Racusin, J. L., Burrows, D. N., Mangano, V., Sakamoto, T., \& Gehrels, N., 2005, GCN Circular 3268

Krimm, H., Barbier, L., Barthelmy, S., Cummings, J., Fenimore, E., Gehrels, N., Hullinger, D., Lamb, D. Q., et al., 2005, GCN Circular 3183

Kouveliotou, C., Meegan, C. A., Fishman, G. J., Bhat, N. P., Briggs, M. S., Koshut, T. M., Paciesas, W. S., \& Pendleton, G. N., 1993, ApJ, 413, L101

Liang, E., \& Zhang, B., 2005, ApJ, in press, astro-ph/0504404i

Mangano, V., La Parola, V., Cusumano, G., Mineo, T., Campana, S., Capalbi, M., Chincarini, G., Giommi, P., et al., 2006, ApJ, submitted, astro-ph/0603738

Nousek, J. A., Kouveliotou, C., Grupe, D., Page, K., Granot, J., Ramirez-Ruiz, E., Patel, S. K., Burrows, D. N., et al., 2006, ApJ, in press, astro-ph/0508332

Panaitescu, A., \& Kumar, P., 2002, ApJ, 571, 779

Patat, F., \& Piemonte, A., 1998, IAUC 6918

Pei, Y., 1992, ApJ, 395, 130

Peng, F. Königl, A., \& Granot, J., 2005, ApJ, 626, 966

Perna, R., Lazzati, D., \& Fiore, F., 2003, ApJ, 585, 775

Predehl, P., \& Schmitt, J. H. M. M., 1995, A\&A, 293, 889

Rhoads, J. E., 1999, ApJ, 525, 737

Roming, P. W. A., Kennedy, T. E., Mason, K. O., Nousek, J. A., Ahr, L., Bingham, R. E., Broos, P. S., Carter, M. J., et al., 2005, Sp. Sci. Rev., 120, 95

Sakamoto, T., Barbier, L., Barthelmy, S. D., Burrows, D. N., Chester, M., Cummings, J., Fenimore, E., Gehrels, N., et al., 2005a, GCN Circular 3264 
Sakamoto, T., Barbier, L., Barthelmy, S. D., Cummings, J. R, Fenimore, E., Gehrels, N., Hullinger, D., Krimm, H., et al., 2005b, GCN Circular 3273

Sakamoto, T., Barbier, L. Barthelmy, S. D., Cummings, J. R., Fenimore, E. E., Gehrels, N., Hullinger, D., Krimm, H. A., et al., 2006, ApJ, 636, L73,

Sari, R., Piran, T., \& Halpern, J. P., 1999, ApJ, 519, L17

Schady, P., Sakamoto, T., McGowan, K., Boyd, P., Roming, P., Nousek, J. \& Gehrels, N., 2005a, GCN Circular 3276

Schady, P., Sakamoto, T., McGowan, K., Boyd, P., Roming, P., Nousek, J. \& Gehrels, N., 2005b, GCN Circular 3280

Schady, P., Mason, K. O., Osborne, J. P., Page, M. J., Roning, P. W. A., Still, M., Zhang, B., Blustin, A. J., et al., 2006, AJ, submitted, astro-ph/0601182

Schlegel, D. J., Finkbeiner, D. P., \& Davis, M., 1998, ApJ, 500, 525

Sheth, K., Frail, D. A., White, S., Das, M., Bertoldi, F., Walter, F., Kulkarni, S. R., \& Berger, E., 2003, ApJ, 595, L33

Skrutskie, M. F., Cutri, R. M., Stiening, R., Weinberg, M. D., Schneider, S., Carpenter, J. M., Beichman, C., Capps, R., et al., 2006, AJ, 131, 1163

Soderberg, A. M., 2005, GCN Circular 3318

Stratta, G., Fiore, F., Antonelli, L. A., Piro, L., \& De Pasquale, M., 2004, ApJ, 608, 846

Waxman, E., \& Draine, B. T., 2000, ApJ, 537, 796 